\title{
ADIÇÃO DE GESSO AGRÍCOLA EM TRÊS TIPOS DE CAMA DE AVIÁRIO NA FIXAÇÃO DE NITROGÊNIO E NO DESEMPENHO DE FRANGO DE CORTE ${ }^{1}$
}

\author{
ADDITION OF CALCIUM SULFATE IN THREE TYPES OF LITTER \\ ABOUT THE FIXATION OF NITROGEN AND \\ ON BROILER PERFORMANCE
}

\author{
Rafael Neme $^{2}$ Nilva Kazue Sakomura ${ }^{3}$ Mauro Dal Seco de Oliveira ${ }^{3}$ \\ Flavio Alves Longo ${ }^{4}$ Adriana Nogueira Figueiredo ${ }^{2}$
}

\section{RESUMO}

O experimento foi conduzido com o objetivo de avaliar os efeitos da adição de gesso agrícola (Sulfato de Cálcio) em três tipos de cama de aviário sobre o desempenho de frango de corte e fixação de nitrogénio na cama. Foram utilizadas 1440 aves da linhagem Hubbard, submetidas a um delineamento inteiramente casualizado. Os tratamentos foram distribuídos em esquema fatorial ( $3 \times 2 \times 2$ ) para testar três tipos de cama (casca de arroz, maravalha e casca de amendoim), adição ou não de gesso e sexo, sendo 12 tratamentos com 3 repetições de 40 aves cada. Em cada parcela, foi colocado $35 \mathrm{~kg}$ de cama e nas parcelas com gesso foram adicionados e incorporados $15 \mathrm{~kg}$ deste material (43\%). O desempenho das aves foi avaliado aos 21, 38 e 45 dias de idade. Ao final do experimento, foram coletadas amostras das camas para análise de $\mathrm{pH}$, matéria seca e nitrogénio. Os resultados demonstraram que a adição de gesso agrícola não influenciou o desempenho das aves e não diminuiu as perdas de nitrogénio das camas, sob forma de amónia. Os diferentes tipos de cama não influenciaram o desempenho das aves, exceto para o consumo de ração de la 21 dias e l a 45 dias de idade, em que as aves mantidas em maravalha apresentaram maior consumo. A adição de gesso agrícola promoveu um decréscimo no valor de pH das camas. Os valores de matéria seca e nitrogénio não diferiram para os diferentes tipos de camas e para a adição ou não de gesso. Em relação ao sexo, foram observadas diferenças para o teor de nitrogénio das camas, sendo maior para machos devido, principalmente, a maior produção de excretas destes.

Palavras-chave: cama de frango, fixação de nitrogénio, frangos de corte, gesso agrícola.

\section{SUMMARY}

An experiment was conducted to determine the effects of the addition or not of calcium sulfate and three differents types of litter (rice seed coat. wood shaver and peanut seed coat). on the $\mathrm{pH}$, dry matter and nitrogen fixation in the litter and on the broiler performance. A randomized complete design with a factorial arrangement of $3 \times 2 \times 2$ \{three different type of litter, two levels of calcium sulfate and two sex) was used with three replicates ( $n=1440: 40$ chicks per replicate) per treatment. In each treatment was used $35 \mathrm{~kg}$ of litter and $O$ or $15 \mathrm{~kg}$ of calcium sulfate. The broiler performance was evaluated at 21, 38 and 45 days of age. Samples of litter were coilected at the end of the experiment. The results show that the addition or not of calcium sulfate did not influence the performance of the birds and did not decrease the lose of nitrogen through ammonia volatilization, but decreased the $\mathrm{pH}$ in the litter. The litter the differents types of materiais used in the litter did not influence the broilers performance. However, when the wood shaver was used, the feed in take was higher. The dry matter and nitrogen were not affected by any of the parameters studied. The different types of material and levels of calcium sulfate used in the litter did not affèct the dry matter and nitrogen content. The nitrogen content where the inale were raised was higher than the litter of the females. This may be affected by the higher manure production by the males.

Key words: broiler chickens, calcium sulfate, fixation of nitrogen, litter.

\footnotetext{
${ }^{1}$ Trabalho apresentado pelo primeiro autor à Faculdade de Ciências Agrárias e Veterinárias da Universidade do Estado de São Paulo (FCAV/UNESP), Jaboticabal, para Graduação em Zootecnia.

${ }^{2}$ Zootecnista formada pela FCAV/UNESP - Jaboticabal.

${ }^{3}$ Professores do Departamento de Zootecnia, FCAV/UNESP, 14884-900, Jaboticabal. E-mail: sakomura@fcav.unesp.br. Autor para correspondência.

${ }^{4}$ Estudante de Pós-Graduação em Produção Animal, FCAV/UNESP, Jaboticabal. 


\section{INTRODUÇÃO}

A cama de aviário é rica em nitrogênio não proteico, principalmente na forma de ácido úrico, e quando oferecida na alimentação de ruminantes é utilizada na síntese de proteína. ANDRIGUETTO (1981) observou que as camas avícolas possuem em torno de $2440 \mathrm{kcal}$ de energia digestível e proteína bruta variando de 18 a 40\%, se considerados na matéria seca. Devido à ação de microorganismos decompositores de compostos nitrogenados ocorre a fermentação da cama com consequente perda de nitrogênio por volatilização da amônia $\left(\mathrm{NH}_{3}\right.$, aumentando sua concentração no ambiente, o que, além de ser prejudicial ao desempenho das aves, diminui o valor nutritivo dessa fonte de proteína. Uma alternativa estudada para diminuir as perdas de nitrogénio por volatilização da amônia é a adição de algumas substâncias ou compostos à cama que favorecem através de reações químicas o aumento na fixação do nitrogénio. Uma das substâncias ou compostos estudados para esse fim é o sulfato de cálcio $\left(\mathrm{CaSO}_{4}\right)$, também conhecido como gesso agrícola. Segundo MALA VOLTA $\boldsymbol{e t}$ al. (1979), durante o processo de fermentação do esterco perde-se até $60 \%$ do nitrogênio na forma de amônia em um período de quatro meses. GLÓRIA et al. (1991) avaliaram o gesso agrícola e outros produtos como inibidores da volatilização de amónia de três tipos de estercos; dentre eles, a cama de frango apresentou valores intermediários nas perdas de nitrogénio em relação ao esterco puro de galinhas poedeiras e ao estercos de bovinos em confinamento em mistura com bagaço de cana. O superfosfato simples foi melhor que o gesso, inibindo 58,9 e $39,3 \%$ das perdas de nitrogénio respectivamente, sendo que os outros materiais testados não apresentaram ação inibidora.

$\mathrm{O} \mathrm{pH}$ da cama tem um papel importante na volatilização de amónia. Uma vez formada, a amónia livre estará em uma das duas formas: $\mathrm{NH}_{3}$ sem carga ou na forma de íon amónia $\left(\mathrm{NH}_{4}\right)$, dependendo do $\mathrm{pH}$ da cama (IVOS et al., 1966). Segundo CARR (1990), a concentração de amónia aumenta com o aumento do $\mathrm{pH}$. A liberação de amónia é menor quando o pH da cama está abaixo de 7,0, mas é substancial quando está acima de 8,0, sendo que a decomposição do ácido úrico é mais favorecida em condições de pH alcalino (TERZICH, 1997). Na literatura, verifica-se uma variação nos valores de $\mathrm{pH}$ da cama de frango atribuída à metodologia de análise. Os valores médios de $\mathrm{pH}$ encontrados por SAMPAIO et $\boldsymbol{a l}$. (1997), ao analisarem a adição de gesso à cama de maravalha em adição única ou adição parcelada, foram, respectivamente, 6,99 e 6,98.
JORGE et al. (1990) encontraram no final do experimento $\mathrm{pH}$ de 7,5 em cama de maravalha e 7,8 em cama tratada com calcário dolomítico.

A umidade da cama de frangos varia muito em função do manejo adotado e, segundo MIELE \& MILAN (1983), pode variar de 26,5 a $67,8 \%$. SAMPAIO et al. (1997) encontraram valores de umidade em camas de frango com adição de gesso variando entre $14,54 \%$ e $19,00 \%$, indicando que a adição de gesso promove uma menor umidade da cama.

Em relação aos tipos de materiais usados como cama de aviário, a maravalha é um material proveniente do beneficiamento da madeira, apresentando partículas de tamanho aproximado de $3 \mathrm{~cm}$, com um bom poder de absorção, sendo o material mais utilizado na avicultura. ÁVILA et al. (1992) comentaram que a casca de arroz apresenta certas restrições ao seu uso, devido à baixa capacidade de absorção e por ser composta de partículas pequenas que podem ser ingeridas pelos pintos em demasia acarretando riscos de intoxicação.

A casca de amendoim é um material que apresenta propriedades absorventes, de boa compressão e homogeneidade, restringido-se o uso, no caso de excesso de umidade, pois pode vir a apresentar contaminação pelo fungo Aspergilius flavus ou Aspergilius fumigatus, levando as aves a um quadro de aspergilose.

Devido à necessidade do melhor manejo da cama aviária para a obtenção de um subproduto de boa qualidade para a alimentação animal, este trabalho teve por objetivo verificar os efeitos da adição de gesso agrícola em camas de maravalha, casca de arroz e casca de amendoim sobre o desempenho de frangos de corte e fixação de nitrogênio.

\section{MATERIAL E MÉTODOS}

O experimento foi realizado no Aviário experimental do departamento de Zootecnia da Faculdade de Ciências Agrárias e Veterinárias Jaboticabal - SP, no período de março a maio de 1997. Utilizaram-se 1440 pintos de corte com um dia de idade, da linhagem Hubbard. O delineamento experimental utilizado foi o inteiramente casualizado, com distribuição dos tratamentos em esquema fatorial ( $3 \times 2 \times 2)$, sendo estudados os fatores: três tipos de cama, adição ou não de gesso agrícola e sexo das aves, totalizando 12 tratamentos com três repetições de 40 aves cada. Foram testadas três camas de aviário: casca de arroz, casca de amendoim e maravalha, sendo colocado $35 \mathrm{~kg}$ de material por parcela. Nas parcelas com gesso, foram adicionados e incorporados $15 \mathrm{~kg}$ deste material (43\%). As camas foram 
revolvidas semanalmente para melhor incorporação. Os tratamentos utilizados foram: $\mathrm{T}_{1}$-Casca de arroz, $\mathrm{T}_{2}$-Maravalha, $\mathrm{T}_{3}$-Casca de amendoim, $\mathrm{T}_{2}$-Casca de arroz + gesso, $\mathrm{T}_{5}$-Maravalha + gesso, $\mathrm{T}_{6}$ - Casca de amendoim + gesso, para cada sexo. As rações experimentais foram formuladas para atender as exigências nutricionais das aves de acordo com o manual da linhagem em cada uma das fases de criação (inicial, crescimento e terminação). Foram estudados parâmetros de desempenho das aves, como consumo de ração, peso das aves e conversão alimentar. Para a avaliação das camas foram determinados os teores de matéria seca, nitrogénio, $\mathrm{pH}$, produção total de cama por $\mathrm{m}^{2}$ e por ave. A análise estatística foi feita através do programa ESTAT desenvolvido no Departamento de Ciências Exatas da UNESP - Jaboticabal, e as médias foram comparadas pelo teste Tukey.

\section{RESULTADOS E DISCUSSÃO}

Na tabela 1, observa-se que não houve interação significativa entre tipos de cama, adição de gesso e sexo para o consumo de ração; houve diferença significativa para o consumo de ração na fase de 01 a 21 dias, em que as aves criadas em cama de maravalha apresentaram maior consumo de ração em relação aos demais tratamentos. Nessa fase, o menor consumo de ração das aves criadas nas camas com casca de arroz e casca de amendoim provavelmente ocorreu devido à ingestão de pequenas partículas presentes nessas camas.

No período de 01 a 45 dias, houve diferença significativa no consumo de ração entre as aves criadas em cama de maravalha e casca de amendoim. Esses resultados discordam de BARRIGA et al. (1970), os quais não encontraram diferenças significativas para consumo de ração de aves criadas em diferentes tipos de cama de aviário. Nas fases de 22 a 38 e 39 a 45 dias, não houve diferença significativa entre os tratamentos. A adição de gesso não influenciou o consumo de ração das aves, concordando com LON-WO \& RODRIGUEZ (1986), que verificaram a utilização de cal (óxido de cálcio), semelhante ao gesso, em camas de aviário para a fixação de nitrogênio, não encontrando diferenças significativas para consumo de ração. Nas três fases e no período total de criação das aves, os machos tiveram maior consumo de ração que as fêmeas, como era esperado.

Analisando a tabela 2, não se observou interação significativa entre tipos de cama, adição de gesso e sexo, assim como não se obteve diferenças significativas nas diferentes fases de criação entre os tipos de cama e a adição de gesso à cama para o peso das aves. Podem-se observar diferenças
Tabela 1 - Análise de variância e médias de consumo de ração (kg/ave) nas diferentes fases de criação de frango de corte.

\begin{tabular}{|c|c|c|c|c|}
\hline & \multicolumn{4}{|c|}{ IDADE EM DIAS } \\
\hline Fatores & $1-21$ & $22-38$ & $39-45$ & $1-45$ \\
\hline Casca de arroz & $0,974^{\mathrm{b}}$ & 2,326 & 1,243 & $4,519^{\mathrm{ab}}$ \\
\hline Maravalha & $1,005^{\mathrm{a}}$ & 2,346 & 1,266 & $4,578^{\mathrm{a}}$ \\
\hline Casca de amendoim & $0,974^{b}$ & 2,264 & 1,202 & $4,414^{b}$ \\
\hline Com gesso & 0,986 & 2,324 & 1,240 & 4,525 \\
\hline Sem gesso & 0,983 & 2,300 & 1,234 & 4,483 \\
\hline Macho & $1,018^{\mathrm{a}}$ & $2,455^{\mathrm{a}}$ & $1,351^{a}$ & $4,764^{a}$ \\
\hline \multirow[t]{2}{*}{ Fêmea } & $0,950^{\mathrm{b}}$ & $2,170^{\mathrm{b}}$ & $1,1225^{\mathrm{b}}$ & $4,244^{b}$ \\
\hline & \multicolumn{4}{|c|}{ Valores de F } \\
\hline Cama (C) & $4,3581 *$ & $1,9563^{\mathrm{ns}}$ & $3,1614^{\mathrm{ns}}$ & $3,5092 *$ \\
\hline Gesso (G) & $0,1207^{\mathrm{ns}}$ & $0,4559^{\mathrm{ns}}$ & $0,0739^{\mathrm{ns}}$ & $0,6838^{\mathrm{ns}}$ \\
\hline $\operatorname{Sexo}(\mathrm{S})$ & $47,0533^{* *}$ & $64,8922 * *$ & $118,0028 * *$ & $103,9736^{* *}$ \\
\hline Interação (CxG) & $0,0298^{\mathrm{ns}}$ & $0,9579^{\text {ns }}$ & $0,3260^{\mathrm{ns}}$ & $0,6411^{\mathrm{ns}}$ \\
\hline Interação (CxS) & $1,1364^{\mathrm{ns}}$ & $0,6422^{\mathrm{ns}}$ & $0,1734^{\mathrm{ns}}$ & $0,1796^{\mathrm{ns}}$ \\
\hline Interação (GxS) & $0,0363^{\mathrm{ns}}$ & $0,1933^{n s}$ & $0,3400^{n s}$ & $1,3667^{\mathrm{ns}}$ \\
\hline Interação (CxGxS) & $0,3125^{\mathrm{ns}}$ & $0,1274^{\mathrm{ns}}$ & $0,7580^{\mathrm{ns}}$ & $0,0978^{\text {ns }}$ \\
\hline C.V (\%) & 3,02 & 4,59 & 5,11 & 3,40 \\
\hline
\end{tabular}

Médias seguidas de letras diferentes sobrescritas na mesma coluna diferem entre si pelo teste Tukey.

* Significativo em nível de $5 \%$ de probabilidade.

** Significativo em nível de $1 \%$ de probabilidade. ns Não significativo.

significativas apenas para o fator sexo em todas as fases de criação, como era esperado. Esses resultados indicaram que os tipos de cama avaliados não interferiram no peso das aves. Resultados semelhantes foram observados por MOUCHEREK et al. (1987) e WYATT \& GOODMAN (1992), os quais, avaliando diferentes materiais como cama de aviário e adição de gesso ou não, não observaram diferenças significativas para peso das aves.

Na tabela 3, observa-se que não ocorreram diferenças significativas nas interações entre tipos de cama, adição de gesso e sexo para a conversão alimentar. Verificou-se um efeito significativo para o fator sexo nas fases de 1 a 21 e 22 a 38 dias, assim como no período total de criação das aves. Tal comportamento era esperado, uma vez que a conversão alimentar de fêmeas é pior em relação a de machos. Em relação aos tipos de cama, não se constatou diferenças significativas para conversão alimentar. De acordo com os resultados obtidos por BRAKE et al.(1992), diferentes tipos de cama de 
Tabela 2 - Análise de variância e médias de peso das aves $(\mathrm{kg})$ nas diferentes fases de criação de frangos de corte.

\begin{tabular}{|c|c|c|c|}
\hline & \multicolumn{3}{|c|}{ IDADE EM DIAS } \\
\hline Fatores & 21 & 38 & 45 \\
\hline Casca de arroz & 0,639 & 1,913 & 2,441 \\
\hline Maravalha & 0,663 & 1,957 & 2,485 \\
\hline Casca de amendoim & 0,643 & 1,899 & 2,403 \\
\hline Com gesso & 0,645 & 1,907 & 2,435 \\
\hline Sem gesso & 0,652 & 1,938 & 2,451 \\
\hline Macho & $0,681^{\mathrm{a}}$ & $2,074^{\mathrm{a}}$ & $2,645^{\mathrm{a}}$ \\
\hline \multirow[t]{2}{*}{ Fêmea } & $0,616^{b}$ & $1,772^{b}$ & $2,241^{\mathrm{b}}$ \\
\hline & \multicolumn{3}{|c|}{ Valores de F } \\
\hline Cama (C) & $3,2338^{\text {ns }}$ & $2,6696^{\mathrm{ns}}$ & $2,6956^{\text {ns }}$ \\
\hline Gesso (G) & $0,5788^{\text {ns }}$ & $2,1236^{\mathrm{ns}}$ & $0,2705^{\mathrm{ns}}$ \\
\hline $\operatorname{Sexo}(S)$ & $59,7205^{* *}$ & $200,5053^{* *}$ & $193,3231 * *$ \\
\hline Interação (CxG) & $1,3986^{\mathrm{ns}}$ & $0,4227^{\text {ns }}$ & $0,6817^{\text {ns }}$ \\
\hline Interação (CxS) & $0,0914^{\mathrm{ns}}$ & $0,1578^{\mathrm{ns}}$ & $0,0349^{\text {ns }}$ \\
\hline Interação (GxS) & $1,1401^{\mathrm{ns}}$ & $0,2951^{\text {ns }}$ & $0,7078^{\text {ns }}$ \\
\hline Interação (CxGxS) & $0,1739^{\text {ns }}$ & $0,0484^{n s}$ & $0,2119^{\text {ns }}$ \\
\hline C.V (\%) & 3,85 & 3,32 & 3,57 \\
\hline
\end{tabular}

Médias seguidas de letras diferentes sobrescritas na mesma coluna diferem entre si pelo teste Tukey.

** Significativo em nível de $1 \%$ de probabilidade ns Não significativo.

aviário não afetam significativamente a conversão alimentar de frangos de corte. NAKAUE $\boldsymbol{e t} \boldsymbol{a l}$. (1978) também não encontraram diferenças significativas para a conversão alimentar com e sem adição de gesso.

Com relação às características da cama aviária, observa-se na tabela 4 que os teores médios de matéria seca das camas utilizadas não foram afetados significativamente pêlos fatores estudados. Os valores de matéria seca das camas variaram de 69 a $73 \%$ e foram maiores que os encontrados por BRAKE et al. (1992), os quais verificaram teores de matéria secas em média de $60 \%$; contudo, foram menores que os valores encontrados por JORGE $\boldsymbol{e t}$ al. (1990), de 83,42\% para cama de maravalha e $82,42 \%$ para cama tratada com calcário dolomítico. SAMPAIO et al. (1997) observaram valores médios de matéria seca de camas de frango tratadas com gesso, variando de 81,00 a $85,46 \%$.

Observa-se na tabela 4 que a adição de gesso às camas promoveu uma queda no $\mathrm{pH}(8,11)$ em relação às camas sem gesso $(8,96)$. SAMPAIO $\boldsymbol{e t}$ al. (1997) também encontraram $\mathrm{pH}$ mais baixo
$(6,89)$ em camas com adição de gesso agrícola que o $\mathrm{pH}$ de camas isentas de gesso $(7,06)$. HUFF $\boldsymbol{e t}$ al.(1984) encontraram, ao final de sete semanas de criação das aves, valores de $\mathrm{pH}$ em torno de 8,0; valor semelhante ao encontrado neste trabalho. Houve interação significativa entre tipos de camas e sexo. Para machos não houve diferença significativa entre os tipos de cama, mas para as fêmeas o pH da cama com casca de amendoim foi significativamente superior ao $\mathrm{pH}$ da cama de maravalha, sendo que o pH da cama de casca de arroz não diferiu significativamente dessas camas. A cama com maravalha onde as fêmeas foram criadas apresentou menor valor de $\mathrm{pH}$, diferindo estatisticamente dos machos.

Segundo TERZICH (1997), a liberação de amónia é menor quando o $\mathrm{pH}$ da cama está abaixo de 7,0, mas é maior quando está acima de 8,0. Uma das principais bactérias ureolíticas, Bacilius pasteurii, não consegue crescer em $\mathrm{pH}$ neutro, mas prospera na cama com $\mathrm{pH}$ acima de 8,5. Verificouse, no presente trabalho, que a adição de gesso agrícola à cama de frango reduziu o $\mathrm{pH}$ das camas, o aviário

Tabela 3 - Análise de variância e médias de conversāo alimentar das aves nas diferentes fases de criação de frangos de

\begin{tabular}{|c|c|c|c|c|}
\hline & \multicolumn{4}{|c|}{ IDADE EM DIAS } \\
\hline Fatores & $1-21$ & $22-38$ & $39-45$ & $1-45$ \\
\hline Casca de arroz & 1,63 & 1,78 & 2,36 & 1,89 \\
\hline Maravalha & 1,62 & 1,77 & 2,40 & 1,88 \\
\hline Casca de amendoim & 1,62 & 1,75 & 2,39 & 1,90 \\
\hline Com gesso & 1,64 & 1,79 & 2,35 & 1,90 \\
\hline Sem gesso & 1,61 & 1,74 & 2,42 & 1,88 \\
\hline Macho & $1,59^{\mathrm{h}}$ & $1,72^{b}$ & 2,37 & $1,86^{\mathrm{b}}$ \\
\hline \multirow[t]{2}{*}{ Fêmea } & $1,65^{\mathrm{a}}$ & $1,81^{\mathrm{a}}$ & 2,40 & $1,94^{\mathrm{a}}$ \\
\hline & \multicolumn{4}{|c|}{ Valores de F } \\
\hline Cama (C) & $0,2191^{\mathrm{ns}}$ & $0,3164^{n s}$ & $0,5820^{\mathrm{ns}}$ & $0,1037^{\mathrm{ns}}$ \\
\hline Gesso (G) & $1,4688^{\text {ns }}$ & $4,1194^{n s}$ & $2,7642^{n s}$ & $2,4059^{\mathrm{ns}}$ \\
\hline Sexo $(S)$ & $8,3593^{* *}$ & $17,5520^{* *}$ & $0,6082^{\text {ns }}$ & $72,8313^{* *}$ \\
\hline Interação (CxG) & $1,5127^{\text {ns }}$ & $1,1707^{\text {ns }}$ & $1,7693^{\text {ns }}$ & $0,4630^{\text {ns }}$ \\
\hline Interação (CxS) & $1,1891^{\mathrm{ns}}$ & $2,1938^{n s}$ & $0,6875^{\text {ns }}$ & $1,5262^{\mathrm{ns}}$ \\
\hline Interação (GxS) & $1,1231^{\mathrm{ns}}$ & $0,0025^{\text {nis }}$ & $0,6128^{\text {ns }}$ & $0,8029^{\text {ns }}$ \\
\hline Interaçāo (CxGxS) & $0,1087^{\mathrm{ns}}$ & $0,7368^{\mathrm{ns}}$ & $0,2674^{n s}$ & $0,6995^{n s}$ \\
\hline C.V $(\%)$ & 3,78 & 3,99 & 4,69 & 1,42 \\
\hline
\end{tabular}

Médias seguidas de letras diferentes sobrescritas na mesma coluna diferem entre si pelo teste Tukey.

** Significativo em nível de $1 \%$ de probabilidade. ns Não significativo. 
Tabela 4 - Análise de variância para matéria seca (MS \%), pH e nitrogênio ( $\mathrm{N} \%$ ) das camas ao final do experimento.

\begin{tabular}{|c|c|c|c|c|}
\hline & \multicolumn{4}{|c|}{ PARÂMETROS AVALIADOS } \\
\hline \multirow[t]{2}{*}{ Fatores } & \multirow[t]{2}{*}{$\operatorname{MS}(\%)$} & \multicolumn{2}{|c|}{$\mathrm{pH}$} & \multirow[t]{2}{*}{$N(\%)$} \\
\hline & & Macho & Fêmea & \\
\hline Casca de arroz & 72,78 & $8,58^{\mathrm{aA}}$ & $8,54^{\mathrm{abA}}$ & 2,79 \\
\hline Maravalha & 72,93 & $8,61^{\text {aA }}$ & $8,38^{\mathrm{bB}}$ & 2,94 \\
\hline Casca de amendoim & 69,02 & $8,45^{\text {aA }}$ & $8,66^{\mathrm{aA}}$ & 2,88 \\
\hline Com gesso & 71,04 & \multicolumn{2}{|c|}{$8,11^{\mathrm{b}}$} & 2,89 \\
\hline Sem gesso & 72,12 & \multicolumn{2}{|c|}{$8,96^{\mathrm{a}}$} & 2,84 \\
\hline Macho & 70,90 & \multicolumn{2}{|c|}{8,55} & $2,95^{\mathrm{a}}$ \\
\hline Fêmea & 72,25 & \multicolumn{2}{|c|}{8,53} & $2,78^{\mathrm{b}}$ \\
\hline & \multicolumn{4}{|c|}{ Valores de F } \\
\hline Cama (C) & $3,9339 *$ & \multicolumn{2}{|c|}{$0.5214^{\mathrm{ns}}$} & $1,1888^{\mathrm{ns}}$ \\
\hline Gesso (G) & $0,7064^{\text {ns }}$ & \multicolumn{2}{|c|}{$231,5565^{* *}$} & $0,3393^{\mathrm{ns}}$ \\
\hline $\operatorname{Sexo}(S)$ & $1,1063^{\text {ns }}$ & \multicolumn{2}{|c|}{$0,1016^{\mathrm{ns}}$} & $4,4663^{*}$ \\
\hline Interação (CxG) & $1,9777^{\mathrm{ns}}$ & \multicolumn{2}{|c|}{$1,0587^{\text {ns }}$} & $0,7852^{\text {ns }}$ \\
\hline Interação (CXS) & $0,7847^{\mathrm{ns}}$ & \multicolumn{2}{|c|}{$5,1254^{*}$} & $0,8750^{\mathrm{ns}}$ \\
\hline Interação (GxS) & $3,7209^{\mathrm{ns}}$ & \multicolumn{2}{|c|}{$0,7680^{\mathrm{ns}}$} & $2,6428^{\mathrm{ns}}$ \\
\hline Interação (CxGxS) & $1,1251^{\text {ns }}$ & \multicolumn{2}{|c|}{$0,1024^{\mathrm{ns}}$} & $0,3470^{\mathrm{ns}}$ \\
\hline C.V $(\%)$ & 5,40 & \multicolumn{2}{|c|}{1,96} & 8,58 \\
\hline
\end{tabular}

Médias seguidas de letras diferentes sobrescritas na mesma coluna diferem entre si pelo teste Tukey.

* Significativo em nível de $5 \%$ de probabilidade.

** Significativo em nível de $1 \%$ de probabilidade.

ns Não significativo.

que proporcionou condições desfavoráveis para o crescimento das bactérias ureolíticas, reduzindo portanto, a decomposição do ácido úrico e, conseqüentemente, a volatilização do nitrogénio na forma de amónia.

Os tipos de cama e a adição ou não de gesso agrícola não influenciaram os teores de nitrogénio das camas no final da criação, assim como não houve interações entre tipos de cama, adição ou não de gesso e sexo (Tabela 4). A média dos teores de nitrogênio dos três tipos de cama e adição ou não de gesso agrícola foi de 2,87\%, semelhante à média $(3,02 \%)$ obtida em vinte e cinco galpões de frangos de corte estudados por MIELE \& MILAN (1983). Houve diferença significativa nos teores de nitrogênio das camas para sexo. Os teores de nitrogénio das camas dos machos foram superiores aos teores de nitrogénio das camas das fêmeas. Os maiores teores de nitrogénio nas camas dos machos podem ser explicados pela maior quantidade de excretas produzidas por estes durante o ciclo de criação.
Tabela 5 - Peso médio final de cama em quilogramas de matéria seca produzidos por parcela $\left(4,48 \mathrm{~m}^{2}\right)$, por $\mathrm{m}^{2}$ de cama e por ave, para cada tratamento testado,

\begin{tabular}{ccccc}
\hline & \multicolumn{4}{c}{ PESO DA CAMA kg (MS) } \\
\cline { 3 - 5 } Tipo de cama & $\begin{array}{c}\text { Adição de } \\
\text { gesso }\end{array}$ & $\begin{array}{c}\text { Total } \\
\left(4,48 \mathrm{~m}^{2}\right)\end{array}$ & Por $\mathrm{m}^{2}$ & Por ave \\
& Sim & 77,13 & 17,22 & 1,940 \\
Maravalha & Não & 72,23 & 16,12 & 1,835 \\
Casca de arroz & Sim & 80,90 & 18,06 & 2,032 \\
& Não & 71,13 & 15,88 & 1,812 \\
Casca de amendoim & Sim & 74,69 & 16,67 & 1,885 \\
& Não & 63,45 & 14,16 & 1,632 \\
\hline
\end{tabular}

De acordo com os dados de produção de cama apresentados na tabela 5 , pode-se observar que entre os tipos de cama sem a adição de gesso agrícola houve uma maior produção de cama por ave em camas com maravalha, sendo esta 1,25 e $11,06 \%$ maior do que a produção de cama por ave nas camas com casca de arroz e casca de amendoim, respectivamente. Com a adição de gesso agrícola às camas $(15 \mathrm{~kg})$, a produção de cama por ave, no final da criação das aves, aumentou $5,41 \%$ em camas com maravalha, 10,83\% em camas com casca de arroz e $13,42 \%$ em camas com casca de amendoim. Este maior aumento na produção das camas com casca de amendoim, pode ser explicado pela textura mais fibrosa deste material, incorporando melhor o gesso, assim como a casca de arroz por apresentar partículas muito pequenas e um grande volume, envolvendo o gesso. A maravalha, devido à sua textura, não incorporou bem o gesso. Contudo, com a adição de gesso agrícola, a cama com casca de arroz apresentou uma maior produção por ave, sendo esta 4,53 e 7,23\% maior que a produção de cama por ave das camas com maravalha e casca de amendoim, respectivamente.

\section{CONCLUSÕES}

Os diferentes materiais utilizados como cama não influenciam o desempenho das aves, podendo ser utilizados na criação de frangos de corte. A adição de $43 \%$ de gesso agrícola às camas, não influencia o desempenho das aves, bem como a qualidade das camas e não promove a fixação de nitrogénio às camas testadas, não justificando, portanto, o seu uso na proporção avaliada. 


\section{REFERENCIAS BIBLIOGRÁFICAS}

ANDRIGUETTO, J.M. Nutrição animal: As bases e os fundamentos da nutrição animal. 4 ed. São Paulo: Nobel, 1981. 395p.

ÁVIlA, V.S., MAZZUCO, H., FIGUEREDO, S. Cama de aviário: materiais, reutilização, uso como alimento e fertilizante. Concórdia : EMBRAPA, 1992. 38p. (Circular técnica, 16).

BARRIGA, F.A., ANDRADE, A.N., LYRA, D.A. Comparação entre vários tipos de cama na criação de frangos de corte. In: REUNIÃO ANUAL DA SOCIEDADE BRASILEIRA DE ZOOTECNIA, 1970. Piracicaba, SP. Anais... Piracicaba: SBZ, 1970. v.7. 130p. p.122-124

BRAKE, J.D., BOYLE, C.R, CHAMBLEE, T.N. Evaluation of the chemical and physical properties ofhardwood bark used as a broiler litter material. Poultry Science, v.71, p.467-472, 1992.

CARR, L.E., WHEATON, F.W., DOUGLAS, L.W. Empirical models to determine ammonia concentrations from broiler chicken litter. Transactions of the American Society of Agricultural Engineers, v.33, n.4, p.1337-1342, 1990

GLÓRIA, N.A., BARRETO, M.C.V., MORAES, C.J., et al Avaliação do gesso e de alguns fosfatos como inibidores da volatilização de amónia de estercos. Revista Brasileira de Ciência do Solo, v.15, n.3, p.297-301, 1991

HUFF, W.E., MALONE, G. W., CHALOUPKA, G.W. Effect of litter treatment on broiler performance and certain litter quality parameters. Poultry Science, v.63,p.2167-2171,1984.

IVOS, J., ASAJ, A., MARJANOVIC, L.J., et al. Contribution to the hygiene of deep litter in the chicken house. Poultry Science, v.45, n.4, p.676-683,1966.

JORGE, M.A., RESENDE, J.S., OLIVEIRA, R.L. Estudo preliminar da presença de Escherichia coli e Staphilococcus $\boldsymbol{s p}$ na cama de galinha tratada com calcário dolomítico. Arq
Neme et al Brás Med Vet Zootec, v.42, n. 1, p.49-56,1990.

LON WO, E., RODRIGUEZ, C. A note on the utilization of zeolite or lime on hay litters for broilers. Cuban Journal of Agricultural Science, v.20, n.3, p.259 - 262,1986.

MALAVOLTA, E., ROMERO, J.P., LIEM, T.H. Gesso agrícola: seu uso na adubação e correção do solo. São Paulo : Ultrafértil, 1979. 32p.

MIELE, A., MILAN, P.A. Composição mineral de cama de aviário de frangos de corte e sua utilização na adubação de vinhedos. Pesquisa Agropecuária Brasileira, v.18, n.7, p.729-733,1983.

MOUCHEREK, E., LINHARES, F., MOULIN, C.H.S., et al. Identificação de materiais de "cama" para frangos de corte, 1 Capins napier e braquiária. In: REUNIÃO ANUAL DA SOCIEDADE BRASILEIRA DE ZOOTECNIA, 1987, Brasília, DF. Anais... Brasília: SBZ, 1987. v.24. 427p. p.368.

NAKAUE, H.S., KOELLIKER, J.K., ARSCOTT, G.H. Effect of chinoptilolite (zeolite) on layer and broiler performances and poultry houses enviroment. Poultry Science, v.57, n.4, p.1175,1978.

SAMPAIO, M.A.P.M.M, SCHOCKEN-ITURRINO, R.P., SAMPAIO, A.A.M., et al. Temperatura, umidade e pH da cama de frangos com a adição de gesso durante o ciclo de criação. In: REUNIÃO ANUAL DA SOCIEDADE BRASILEIRA DE ZOOTECNIA, 1997, Juiz de Fora, MG. Anais... Juiz de Fora : SBZ, 1997 (CD Rom).

TERZICH, M.A amónia dos galpões avícolas e o pH da cama. In: CONFERÊNCIA AFINCO DE CIÊNCIA E TECNOLOGIA AVÍCOLAS, 1997, São Paulo, SP. Anais... São Paulo : Associação Brasileira dos Produtores de pintos de Corte, 1997. 304p. p.141-146.

WYATT, C.L., GOODMAN, T.N. Research note: The utilization of recycled sheetrock (refined gypsum) as a litter material for broiler houses. Poultry Science, v.72, n.8, p.1572-1576, 1992.

Ciência Rural, v. 30, n. 4, 2000. 